\title{
Association between anxious distress in a major depressive episode and bipolarity
}

This article was published in the following Dove Medical Press journal:

Neuropsychiatric Disease and Treatment

\author{
Hiroko Sugawara' \\ Takahiro Tsutsumi² \\ Ken Inada ${ }^{2}$ \\ Jun Ishigooka ${ }^{2}$ \\ Mamoru Hashimoto' \\ Minoru Takebayashi' ${ }^{1,3}$ \\ Katsuji Nishimura ${ }^{2}$ \\ 'Department of Neuropsychiatry, \\ Faculty of Life Science, Kumamoto \\ University, Kumamoto, Japan; \\ ${ }^{2}$ Department of Psychiatry, Tokyo \\ Women's Medical University Hospital, \\ Tokyo, Japan; ${ }^{3}$ Division of Psychiatry \\ and Neuroscience, Institute for \\ Clinical Research, National Hospital \\ Organization Kure Medical Center, \\ Hiroshima, Japan
}

Purpose: Mixed features in a major depressive episode (MDE) predict bipolar disorder (BD) The mixed features specifier included in the Diagnostic and Statistical Manual of Mental Disorders Fifth Edition (DSM-5) could be restrictive because it excludes the symptoms common to both mania/hypomania and depression, including psychomotor agitation. On the other hand, an anxious distress (ANXD) specifier has also been introduced in the DSM-5, and psychomotor agitation has been defined as a severity of ANXD. In this study, we retrospectively investigated the association between presence of ANXD in an MDE and bipolarity.

Patients and methods: The subjects were patients admitted with an MDE to the Department of Psychiatry at Tokyo Women's Medical University Hospital from December 2014 to March 2016. Eligible patients were older than 20 years of age and met the DSM-5 criteria for major depressive disorder or BD. All data were extracted from medical records. The subjects were grouped according to whether they did or did not have ANXD. The demographics and clinical features of these groups were compared. Severity of illness was evaluated according to the Hamilton Rating Scale for Depression (HRSD) score on admission.

Results: ANXD was present in 31 and absent in 33 of 64 patients with MDE. The HRSD score was significantly higher in the group with ANXD than in the group without ANXD $(P=0.0041)$. Mixed features $(P=0.0050)$ and suicide attempts $(P=0.0206)$ were significantly more common in the group with ANXD than in the group without ANXD.

Conclusion: We found that the presence of ANXD in an MDE was associated with greater severity and more mixed features and suicide attempts. It is important to evaluate a patient with an MDE for ANXD so that a diagnosis of mixed depression is not missed. More studies in larger samples are needed to investigate further the association between ANXD in MDE and bipolarity. Keywords: anxious distress, mixed features, major depressive episode, bipolarity

\section{Introduction}

Differentiating between bipolar disorder (BD) and major depressive disorder (MDD) is one of the critical tasks in clinical psychiatry, and patients with bipolar depression are at risk of being misdiagnosed with MDD. Five previous studies have investigated potential predictors of BD by multivariate logistic analyses in patients who have had a major depressive episode (MDE).$^{1-5}$ In the previous studies, a family history of BD, onset of a first MDE at a young age, and a history of multiple MDEs were identified as common predictors of BD. Moreover, mixed depression was significantly correlated with BD in three of the five studies. ${ }^{1,2,4}$ Variable definitions of mixed depression were used in some of the studies ${ }^{6,7}$ because of the lack of usefulness of the narrow definition of a mixed episode in the Diagnostic and Statistical Manual of Mental Disorders (DSM)-IV. Therefore, a mixed features specifier was applied in the Diagnostic and Statistical Manual of Mental Disorders Fifth Edition (DSM-5). However, this definition excludes
Correspondence: Hiroko Sugawara Department of Neuropsychiatry, Faculty of Life Science, Kumamoto University, I-I-I Honjo, Chuo-ku, Kumamoto-sh 860-8556, Japan

$\mathrm{Tel}+81963735184$

Fax +81963735184

Email hiroko-sugawara@umin.ac.jp
Neuropsychiatric Disease and Treatment 2019:15 267-270

267

Dovepress f $y$ in 0

http:

(c) (1) (-) 2019 Sugawara et al. This work is published and licensed by Dove Medical Press Limited. The full terms of this license are available at https://www.dovepress.com/terms.php (c)
hereby accept the Terms. Non-commercial uses of the work are permitted without any further permission from Dove Medical Press Limited, provided the work is properly attributed. For permission for commercial use of this work, please see paragraphs 4.2 and 5 of our Terms (https://www.dovepress.com/terms.php). 
the symptoms common to both mania/hypomania and depression, ie, irritability, psychomotor agitation, and distractibility, which are the most common symptoms in mixed depression, from the manic/hypomanic symptoms of an MDE, and there is a possibility of underdiagnosis of mixed depression. ${ }^{8,9}$

However, an anxious distress (ANXD) specifier has also been introduced in the DSM-5, and psychomotor agitation has been defined as a severity of ANXD. Psychomotor agitation in MDD is reportedly related to bipolarity, ${ }^{10}$ and the ANXD specifier could be useful for detection of psychomotor agitation, which was excluded as a manic/hypomanic symptom from the definition of the mixed features specifier in an MDE.

In the present study, we retrospectively investigated the association between the presence of ANXD in an MDE and bipolarity, including family history of MDD/BD, early onset of MDEs, a history of multiple MDEs, and mixed features.

\section{Patients and methods}

\section{Study design}

The study subjects were patients who were admitted with an MDE in the Department of Psychiatry at Tokyo Women's Medical University Hospital from December 2014 to March 2016. Eligible patients were older than 20 years of age and met the criteria for MDD or BD in the DSM-5. All data were extracted from the patients' medical records. We grouped the subjects according to whether or not ANXD was present and compared the demographics (eg, age and sex), diagnosis on admission, clinical features (eg, severity, psychotic symptoms, mixed features, age of onset, duration of current MDE, family history of MDD/BD in a first-degree relative, divorce history, number of recurrent MDE, any suicide attempts, and antidepressant-induced manic episodes), duration of hospitalization, and the prescription at discharge (eg, mood stabilizers, antipsychotics, and antidepressants). Severity of illness was evaluated based on the Hamilton Rating Scale for Depression (HRSD) score on admission. Mixed features were defined according to the criterion used by Benazzi, ie, an MDE with three or more concurrent DSM-IV-defined manic/hypomanic symptoms for more than 1 week. ${ }^{11,12}$

The study was approved by the Department of Psychiatry ethics committee at Tokyo Women's Medical University Hospital and conducted in accordance with the Declaration of Helsinki. Patients were informed about this retrospective study via a bulletin board posting and verbal agreement to participate was secured instead of written informed consent.

\section{Statistical analyses}

Categorical or continuous variables were compared between the two groups using Fisher's exact test or Student's $t$-test, respectively. All statistical analyses were performed using BellCurve for Excel (Social Survey Research Information Co., Ltd.). A $P$-value $<0.05$ was considered statistically significant.

\section{Results}

Thirty-one of 64 patients identified to have had an MDE had ANXD and 33 did not (Table 1). There was no significant

Table I Demographic and clinical variables in the patients with ANXD and without ANXD

\begin{tabular}{|c|c|c|c|}
\hline Demographic and clinical variables & Without ANXD ( $N=33)$ & With ANXD (N=3 I) & $P$-value \\
\hline Age (years) & $52.55 \pm 14.80$ & $56.19 \pm 15.78$ & 0.34 \\
\hline $\operatorname{Sex}(M / F)$ & $10 / 23$ & $5 / 26$ & 0.24 \\
\hline Diagnosis on admission (MDD/BD) & $20 / 13$ & $19 / 12$ & 1.00 \\
\hline HRSD score on admission & $17.04 \pm 8.06$ & $24.03 \pm 8.31$ & $0.004 I^{a}$ \\
\hline Psychosis & $5(15.15 \%)$ & 7 (22.58\%) & 0.53 \\
\hline Mixed features & $4(12.12 \%)$ & $14(45.16 \%)$ & $0.0050^{\mathrm{a}}$ \\
\hline Age of onset (years) & $37.45 \pm 17.42$ & $44.06 \pm 17.89$ & 0.14 \\
\hline Duration of current MDE (months) & $4.59 \pm 10.55$ & $2.32 \pm 2.56$ & 0.25 \\
\hline Family history ${ }^{a}$ & $8(24.24 \%)$ & $7(22.58 \%)$ & 1.00 \\
\hline Divorce history & II (33.33\%) & 7 (22.58\%) & 0.41 \\
\hline Number of recurrent MDE $(>2)$ & $27(81.82 \%)$ & $22(70.97 \%)$ & 0.38 \\
\hline Suicide attempt & $4(12.12 \%)$ & $12(38.71 \%)$ & $0.0206^{\mathrm{a}}$ \\
\hline Antidepressant-induced manic episode & $9(27.27 \%)$ & II (35.48\%) & 0.59 \\
\hline Duration of hospitalization (days) & $59.15 \pm 56.01$ & $75.35 \pm 39.17$ & 0.19 \\
\hline Diagnosis on discharge (MDD/BD) & $17 / 15$ & $17 / 14$ & 1.00 \\
\hline
\end{tabular}

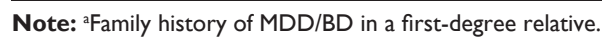

Abbreviations: ANXD, anxious distress; BD, bipolar disorder; HRSD, Hamilton Rating Scale for Depression; MDD, major depressive disorder; MDE, major depressive episode. 
Table 2 The content of prescription on discharge

\begin{tabular}{|l|l|l|}
\hline $\begin{array}{l}\text { Prescription on } \\
\text { discharge }\end{array}$ & $\begin{array}{l}\text { Without ANXD } \\
(\mathbf{N}=\mathbf{3 3})\end{array}$ & $\begin{array}{l}\text { With ANXD } \\
(\mathbf{N}=\mathbf{3} \mathbf{I})\end{array}$ \\
\hline Mood stabilizers & $15(45.45 \%)$ & $18(58.06 \%)$ \\
\hline Antipsychotics & $23(69.70 \%)$ & $15(48.39 \%)$ \\
\hline Antidepressants & $19(57.58 \%)$ & $18(58.06 \%)$ \\
\hline
\end{tabular}

Abbreviation: ANXD, anxious distress.

difference in demographics, diagnosis on admission, or duration of hospitalization between the two groups. The HRSD score was significantly higher in the group with ANXD than in the group without ANXD $(P=0.0041)$. Mixed features $(P=0.0050)$ and attempted suicides $(P=0.0206)$ were significantly more common in the group with ANXD than in the group without ANXD. The results did not change when only subjects with MDD were included (HRSD score, $P=0.0208$; mixed features, $P=0.0084$; attempted suicide, $P=0.0436$ ). The diagnosis was changed from MDD to BD in four patients; half of these patients had ANXD and the diagnosis in one patient without ANXD was changed to somatic symptom disorder.

On discharge, there were no between-group differences in the percentages of psychotropic agents prescribed (Table 2). There was no significant difference in the percentages of lithium prescribed between the patients with ANXD and that without $\operatorname{ANXD}(5 / 18 ; 27.8 \%$ vs $9 / 15 ; 60 \%, P=0.08)$. Regardless of the presence of ANXD, the majority of patients with MDD or BD were prescribed antidepressants (with ANXD, $90 \%$; without ANXD, 89\%) or mood stabilizers (with ANXD, 92\%; without ANXD, 100\%).

\section{Discussion}

In the present study, the prevalence of ANXD in inpatients with an MDE was $48.4 \%$, which is very similar to that in some of the previous studies. ${ }^{13,14}$ However, a higher prevalence of ANXD in MDD was reported in other studies. ${ }^{15,16}$ This lack of concordance may reflect differences in patient's characteristics, including race. In a recent study that investigated the relationship between ANXD and mixed features in Japanese outpatients with MDD or $\mathrm{BD},{ }^{17}$ the prevalence of ANXD was around $30 \%$ in patients with MDD and in those with $\mathrm{BD}$, suggesting that inpatients have more symptoms of anxiety than outpatients. The severity of depression was associated with the presence of ANXD in both the present and previous studies. ${ }^{13,14,17}$ In the present study, mixed features and suicide attempts were significantly more common in the group with ANXD than in the group without ANXD, and these results did not change when only subjects with MDD were analyzed. A previous study reported a higher cumulative incidence of suicide attempts in patients with
BD than in those with MDD, and mixed states contributed to the higher cumulative risk in the BD group. ${ }^{18}$ These findings suggest that some component of bipolarity may be associated with ANXD in an MDE. However, we found that there were no differences in the proportions of patients with MDD or BD between the group with ANXD and the group without ANXD; furthermore, there were no statistically significant differences in some factors representative of bipolarity, ie, family history, early onset of an MDE, and number of recurrent MDEs. These results lead to the fact that the ANXD cannot directly reflect the bipolarity on its own.

Symptoms of anxiety during an MDE may herald a poor response to antidepressants; ${ }^{19}$ however, there are few studies of pharmacotherapy for an MDE with ANXD. The efficacy of adjunctive brexpiprazole treatment for MDD with ANXD has been reported in a post hoc analysis of two randomized controlled studies. ${ }^{20}$ Furthermore, in the mixed depressive patients, antidepressants are not useful or can worsen the depression. ${ }^{21}$ In the present study, regardless of the significant high frequency of mixed features and attempted suicides in patients with ANXD, the prescription on discharge in patients with ANXD was similar to that in patients without ANXD. Especially, there was no significant difference of prescription rate of lithium, which can have anti-suicidal effects, ${ }^{22}$ between the patients with ANXD and without ANXD. The prescription of each main medication was possibly based on the polarity of the diagnosis rather the presence of ANXD that cannot directly reflect the bipolarity.

This study had a retrospective design and the sample size was small, so it is possible that important clinical information was overlooked. However, the quality of all medical record is guaranteed by senior psychiatrists and we carefully extracted each clinical information from the medical record exactly. We could not assess the efficacy of pharmacotherapy because of the retrospective design. In this study, we applied the Benazzi's criteria ${ }^{11,12}$ to define the mixed features; however, there were other criteria such as Koukopoulos' one, ${ }^{23}$ which includes inner tension that overlaps the one of the criteria of ANXD. Our results are needed to validate other criteria of mixed features. Furthermore, we examined only the presence of ANXD according to the criteria of DSM-5, and the severity of ANXD was not evaluated. Considering that psychomotor agitation, which is reportedly related to bipolarity in MDD patients, ${ }^{10}$ has been defined as a severity of ANXD, it is important to investigate the association between ANXD and bipolarity using some clinical scales that can evaluate the severity and discriminate between mixed features and ANXD exactly. 


\section{Conclusion}

We found that the presence of ANXD in an MDE was associated with severe disease, more mixed features, and more suicide attempts. It is important to evaluate ANXD in an MDE so that a diagnosis of mixed depression is not missed. More studies are now needed to investigate more precisely the association between ANXD in MDE and bipolarity and for determination of appropriate pharmacotherapy for ANXD in MDE.

\section{Author contributions}

All of the authors contributed to the conception and design of the study, data analysis, drafting and critically revising the paper, read and approved the manuscript, gave final approval of the version to be published, and agreed to be accountable for all aspects of the work. HS, TT, and KI contributed to data collection. HS performed the statistical analyses and wrote the manuscript.

\section{Disclosure}

The authors report no conflicts of interest in this work.

\section{References}

1. Angst J, Azorin JM, Bowden CL, et al. Prevalence and characteristics of undiagnosed bipolar disorders in patients with a major depressive episode: the bridge study. Arch Gen Psychiatry. 2011;68(8):791-798.

2. Inoue T, Inagaki Y, Kimura T, Shirakawa O. Prevalence and predictors of bipolar disorders in patients with a major depressive episode: the Japanese epidemiological trial with latest measure of bipolar disorder (JET-LMBP). J Affect Disord. 2015;174:535-541.

3. Perlis RH, Brown E, Baker RW, Nierenberg AA. Clinical features of bipolar depression versus major depressive disorder in large multicenter trials. Am J Psychiatry. 2006;163(2):225-231.

4. Takeshima M, Oka T. A comprehensive analysis of features that suggest bipolarity in patients with a major depressive episode: which is the best combination to predict soft bipolarity diagnosis? J Affect Disord. 2013; 147(1-3):150-155.

5. Xiang YT, Zhang L, Wang G, et al. Sociodemographic and clinical features of bipolar disorder patients misdiagnosed with major depressive disorder in China. Bipolar Disord. 2013;15(2):199-205.

6. Swann AC, Lafer B, Perugi G, et al. Bipolar mixed states: an International Society for bipolar disorders Task Force report of symptom structure, course of illness, and diagnosis. Am J Psychiatry. 2013;170(1): $31-42$.

7. Vieta E, Valentí M. Mixed states in DSM-5: implications for clinical care, education, and research. J Affect Disord. 2013;148(1):28-36.
8. Perugi G, Angst J, Azorin JM, et al. Mixed features in patients with a major depressive episode: the BRIDGE-II-MIX study. J Clin Psychiatry. 2015;76(3):e351-e358.

9. Takeshima M, Oka T. DSM-5-defined 'mixed features' and Benazzi's mixed depression: which is practically useful to discriminate bipolar disorder from unipolar depression in patients with depression? Psychiatry Clin Neurosci. 2015;69(2):109-116.

10. Iwanami $\mathrm{T}$, Maeshima $\mathrm{H}, \mathrm{Baba} \mathrm{H}$, et al. Psychomotor agitation in major depressive disorder is a predictive factor of mood-switching. $J$ Affect Disord. 2015;170:185-189.

11. Benazzi F. Which could be a clinically useful definition of depressive mixed state? Prog Neuropsychopharmacol Biol Psychiatry. 2002;26(6): 1105-1111.

12. Benazzi F. Defining mixed depression. Prog Neuropsychopharmacol Biol Psychiatry. 2008;32(4):932-939.

13. Gaspersz R, Lamers F, Kent JM, et al. Longitudinal predictive validity of the DSM-5 anxious distress specifier for clinical outcomes in a large cohort of patients with major depressive disorder. J Clin Psychiatry. 2017;78(2):207-213.

14. McIntyre RS, Woldeyohannes HO, Soczynska JK, et al. The prevalence and clinical characteristics associated with diagnostic and statistical manual Version-5-defined anxious distress specifier in adults with major depressive disorder: results from the International Mood Disorders Collaborative Project. Ther Adv Chronic Dis. 2016;7(3):153-159.

15. Hasin DS, Sarvet al, Meyers JL, et al. Epidemiology of adult DSM-5 major depressive disorder and its specifiers in the United States. JAMA Psychiatry. 2018;75(4):336-346.

16. Zimmerman M, Clark H, McGonigal P, Harris L, Holst CG, Martin J. Reliability and validity of the DSM-5 anxious distress specifier interview. Compr Psychiatry. 2017;76:11-17.

17. Takeshima M. Anxious distress in monopolar and bipolar depression: clinical characteristics and relation with mixed depression in Japan. Psychiatry Clin Neurosci. 2018;72(6):456-457.

18. Holma KM, Haukka J, Suominen K, et al. Differences in incidence of suicide attempts between bipolar I and II disorders and major depressive disorder. Bipolar Disord. 2014;16(6):652-661.

19. Fava M, Rush AJ, Alpert JE, et al. Difference in treatment outcome in outpatients with anxious versus nonanxious depression: a STAR*D report. Am J Psychiatry. 2008;165(3):342-351.

20. Mcintyre RS, Weiller E, Zhang P, Weiss C. Brexpiprazole as adjunctive treatment of major depressive disorder with anxious distress: results from a post-hoc analysis of two randomised controlled trials. J Affect Disord. 2016;201:116-123.

21. Sani G, Napoletano F, Vöhringer PA, et al. Mixed depression: clinical features and predictors of its onset associated with antidepressant use. Psychother Psychosom. 2014;83(4):213-221.

22. Smith KA, Cipriani A. Lithium and suicide in mood disorders: updated meta-review of the scientific literature. Bipolar Disord. 2017;19(7): 575-586.

23. Sani G, Vöhringer PA, Napoletano F, et al. Koukopoulos' diagnostic criteria for mixed depression: a validation study. J Affect Disord. 2014; 164:14-18.
Neuropsychiatric Disease and Treatment

\section{Publish your work in this journal}

Neuropsychiatric Disease and Treatment is an international, peerreviewed journal of clinical therapeutics and pharmacology focusing on concise rapid reporting of clinical or pre-clinical studies on a range of neuropsychiatric and neurological disorders. This journal is indexed on PubMed Central, the 'PsycINFO' database and CAS,

\section{Dovepress}

and is the official journal of The International Neuropsychiatric Association (INA). The manuscript management system is completely online and includes a very quick and fair peer-review system, which is all easy to use. Visit http://www.dovepress.com/testimonials.php to read real quotes from published authors. 